\title{
Digital dermatitis: Natural lesion progression and regression in Holstein dairy cattle over 3 years
}

\author{
Adam C. Krull, ${ }^{*}$ Jan K. Shearer, ${ }^{*}$ Patrick J. Gorden, ${ }^{*}$ H. Morgan Scott, $†$ and Paul J. Plummer* $\ddagger^{1}$ \\ *Department of Veterinary Diagnostic and Production Animal Medicine, College of Veterinary Medicine, lowa State University, Ames 50011 \\ †Department of Veterinary Pathobiology, College of Veterinary Medicine and Biomedical Sciences, Texas A\&M, College Station 77843 \\ ‡Department of Veterinary Microbiology and Preventative Medicine, College of Veterinary Medicine, lowa State University, Ames 50011
}

\begin{abstract}
Bovine digital dermatitis (DD) is a leading cause of lameness in dairy cattle in the United States, with prevalence estimates as high as $30 \%$. Whereas clinical lesions have been well described, little is known about the morphologic changes that are associated with the early stages of lesion development from normal skin to clinical lesions. This study used the Iowa DD scoring system to evaluate the epidemiology of natural lesion development by digitally photographing the rear legs of a cohort of dairy cows over a 3 -yr period. Sixty-one adult Holstein dairy cows were monitored for 1,032 cow foot-months. The incidence rate of lesion development was 4 lesions per 100 cow foot-months, with the average time for a lesion to develop being $133 \mathrm{~d}$. Whereas $20 \%$ of the 1,678 foot observations exhibited clinical DD lesions, an additional $55 \%$ of all observations exhibited preclinical stage 1 and 2 lesions that were indicative of DD lesion development. Utilizing the dichotomous categorization of preclinical lesions in the Iowa DD scoring system, it was found that first-lactation heifers had a higher rate of the thickened and crusted "B" type lesions, whereas the ulcerative "A" type lesions were more likely to be identified in multiparous animals. For clinical DD lesions that received topical treatment, scoring of the post-treatment lesions using the Iowa DD scoring system was found to be useful in prognosticating both the risk of recrudescence and the time until recrudescence. Systemic disease, systemic antibiotic therapy, and periparturient stress were not associated with an increase or decrease in DD lesion scores. Treatment with a single topical tetracycline wrap was associated with a significant decrease $(-1.17)$ in DD lesion score. The results of this study demonstrate that the complex morphologic changes associated with digital dermatitis can be readily classified using the Iowa DD
\end{abstract}

Received October 16, 2015.

Accepted January 12, 2016.

${ }^{1}$ Corresponding author: pplummer@iastate.edu scoring system and the scores can be used to predict and monitor the effects of treatment and prevention measures.

Key words: bovine digital dermatitis, lameness, epidemiology, lesion scoring, dairy cattle

\section{INTRODUCTION}

Bovine digital dermatitis (DD) is a leading cause of lameness among dairy cattle in the United States (USDA, 2009) and appears to be increasing in prevalence among beef cattle (Sullivan et al., 2013). It is estimated that each case of DD costs the farmer between $\$ 95$ to $\$ 133$ in lost production, treatments, and discarded milk (Wilshire and Bell, 2009; Bruijnis et al., 2010; Cha et al., 2010; Fabian et al., 2014). In the most recent National Animal Health Monitoring System (NAHMS) survey of US dairy farms, DD accounted for $61.8 \%$ of the lameness in bred heifers and $49.1 \%$ of the lameness in cows. Estimates of within-herd prevalence have been published across multiple countries (Brown et al., 2000; Holzhauer et al., 2006; Capion et al., 2008, 2009; Cramer et al., 2008; Hulek et al., 2010; van der Linde et al., 2010; Knappe-Poindecker et al., 2013; Becker et al., 2014) and range from $1.4 \%$ in 14 Norwegian herds to $39 \%$ in 5 Danish herds. For herds in freestall barns, most of the estimates for prevalence are in the range of 20 to $25 \%$ (Cramer et al., 2008). However, these estimates are typically based on the prevalence of clinically mature DD lesions that have been described as characteristically circumscribed, erosive to papillomatous, surrounded by a ridge of hyperkeratotic skin, and with hypertrophied hairs (Read and Walker, 1998). This shortcoming (i.e., only considering mature lesions) has resulted in uncertainty regarding the epidemiology of early stage lesions in US dairies. Several authors have published scoring systems designed to describe the progression of DD lesion morphology during the course of lesion development from normal skin to clinical lesions (Döpfer et al., 1997; Manske et al., 2002; Krull et al., 2014). Döpfer et al. (1997) described 4 stages of lesion development with immature DD lesions be- 
ing lumped into a single stage M1 characterized by the formation of a small ulcerative area in the skin. These lesions progress with stage M2 lesions being a mature papillomatous or ulcerative lesion, stage M3 being a healing lesion, and stage M4 being a chronic recurrent lesion (Döpfer et al., 1997). Other scoring systems have been developed to differentiate the variations among active lesions; thus, they are not useful for describing the early stages of lesions (Britt et al., 1999; Manske et al., 2002). To better differentiate the development of early stage lesions, both morphologically and etiologically, our group (Krull et al., 2014) recently described the Iowa DD scoring system. To refine our understanding of lesion development, the Iowa DD scoring system describes 4 distinct morphologies of preclinical lesions that are observed before development of clinical lesions. Each of these preclinical lesion stages, as well as all mature lesion stages, have been shown to contain a unique community of bacteria specific to that lesion stage, thus validating that the morphologic stages can be used as a proxy for the bacterial profile present in the lesion (Krull et al., 2014). The true prevalence for all of these types of DD lesion morphologies in comparison to clinical DD prevalence has yet to be determined.

The treatment of individual animal DD lesions that are causing acute lameness in cattle is important for the clinical management of DD on dairy farms and has typically consisted of topical therapy with tetracycline (Blowey and Sharp, 1988; Hernandez and Shearer, 2000; Manske et al., 2002; Nishikawa and Taguchi, 2008; Cutler et al., 2013) or lincomycin (Laven and Hunt, 2001; Moore et al., 2001; Berry et al. 2012). Most prospective studies designed to evaluate the effectiveness of topical treatments have followed cows for less than 30 d post-treatment. However, clinical experience suggests that treatment success requires long-term follow up of individual animals to observe for lesion regression or recrudescence. Whereas the majority of DD-associated lameness and sensitivity to touch is improved following topical treatment (Cutler et al., 2013), the long-term success of topical treatment resulting in complete healing of the skin on an individual lesion basis has rarely been measured. Berry et al. (2012) followed cattle for $341 \mathrm{~d}$ following topical lincomycin treatment and found that $54 \%$ of all treated lesions required retreatment before the end of the study. Similarly, Read and Walker (1998) showed that $48 \%$ of lesions needed to be retreated within 12 wk following initial treatment. Unfortunately, at present we have no way to determine which lesions are most likely to heal and which are most likely to recrudesce (and hence require retreatment).

Few studies have observed the natural DD lesion development in a controlled environment with the ab- sence of DD prevention strategies (i.e., footbaths). This is largely due to the prolonged period of lesion development, requiring these studies to last for several years, combined with the risk of not utilizing preventative measures. Adding to the complexity of these studies is the difficultly of accurately assessing and documenting lesions over that period of time without closely examining feet by routinely using a hoof trimming chute. An urgent need exists to better understand the natural epidemiology of lesion development from the earliest stages of lesion formation to better understand the potential role of management practices in DD control and prevention. The goal of our study was to follow a group of Holstein cows across several years, including multiple lactations and in the absence of any prevention strategies, to better understand the natural development and progression of DD lesions.

\section{MATERIALS AND METHODS}

Sixty-one adult Holstein dairy cows were enrolled in 3 cohorts over a 3-yr timeframe for this study. The study was conducted at the Iowa State University Dairy Farm (Ames). At the time of the study, the farm was milking approximately 350 cows and the lactating barn consisted of 12 pens with freestalls, mattress liners, and composted manure solids bedding. Cows enrolled during the dry period were housed in a single pen with freestalls and sand bedding. The first cohort of 40 cows consisted of first-, second-, and third-lactation animals that were enrolled as they calved in the summer of 2010. These animals were randomly selected from the herd and enrolled solely based on their calving due date and not being enrolled in any other research trial at the time. This initial cohort of cows was not evaluated for DD status before enrollment and no history of DD lesions was available at the time of enrollment. The second cohort of 13 cows was enrolled in the spring of 2011 and consisted of first-lactation animals that were expected to calve during March and April. These animals were again randomly selected as a convenience sample of animals that were not enrolled in any other research project at that time. In contrast to cohort 1 , this group was screened before enrollment for the presence of DD lesions, and any animal with evidence of classic end-stage DD lesions was excluded from the study. The third cohort of 8 cows was enrolled in the summer of 2012 and consisted only of first-lactation animals that were due during May and June. As with the 2 previous groups, these animals were randomly selected as a convenience sample of animals that were not enrolled in any other research project at that time. This third group was screened before enrollment for the 
presence of DD lesions, and any animal with evidence of either developing or clinical DD lesions was excluded from the study.

Throughout the study, all enrolled cows were excluded from any of the standard DD treatment and prevention procedures used by the Iowa State University dairy. All cows were equipped with radio frequency identification tags which diverted enrolled cows away from the dairy farm's footbath on days in which the rest of the herd was treated. Additionally, all trial cows were marked with unique leg bands to avoid topical parlor treatments and to avoid cows being presented for routine treatments by farm staff or the foot trimmer. Routine foot trimming and treatment of non-DD foot lesions were provided by study personnel to allow for a complete recording of lesion history. Cows were assigned to 1 of the 12 lactating pens based on their lactation number and stage of lactation following the farms standard procedures. As the study was $3 \mathrm{yr}$ in length, cows were allowed to be removed from the study if the farm selected them for culling for any reason. The most common historical reasons for culling from the herd of cows enrolled in this study included reproductive performance and coliform mastitis.

At the time of enrollment, each of the enrolled study cows was lifted on a tilt table for detailed examination of each foot to determine and record evidence of DD lesions. The feet were lightly washed with tap water and each foot was scored using the Iowa DD lesion scoring system (described below) as well as being digitally photographed to allow for blinded lesion scoring using pictures. All cattle enrolled in this study were examined in the same manner every 3 to 4 wk throughout the study period. All observations were made by a single observer to ensure consistency. In addition, full thickness skin biopsies were collected for each lesion when it was observed to change Iowa DD lesion score between observation periods. Biopsies were collected utilizing intravenous regional limb anesthesia with $2 \%$ Lidocaine $\mathrm{HCl}$ (Lidocaine 2\%, Vetone, Boise, ID) and punch biopsies ranging in size from 3 to $6 \mathrm{~mm}$. The feet were lightly washed with tap water to remove all organic debris, but were not scrubbed with any disinfectant such as to minimize the disruption of the natural progression of disease. These biopsies were evaluated by bacterial metagenomics as described in a separate manuscript (Krull et al., 2014). All animal procedures and biopsy methods were approved before the initiation of the study by the Institutional Animal Care and Use Committee of Iowa State University.

Lesion scoring was done utilizing the Iowa DD lesion scoring system, as described previously (Krull et al., 2014). Briefly, this scoring system classifies lesions as either normal skin (stage 0), initial onset lesions (stage 1 ), developing lesions (stage 2), lesions associated with clinical disease in the acute hyperemic ulcerative form (stage 3), or the chronic hyperkeratotic form (stage 4). Stage 1 lesions are characterized as small, localized focal to multifocal lesions that have failed to coalesce into a continuous lesion that involves the majority of the plantar skin adjacent to the interdigital cleft. Once the lesions have coalesced to involve the majority of the plantar skin adjacent to the interdigital cleft they would be characterized as stage 2 . The transition to stage 3 lesions is characterized by the presence of an ulcerated, hyperemic circular lesion that has the roughened strawberry-like appearance of a classical DD lesion. Additionally, stages 1 and 2 are subdivided into 2 subtypes, with "A" type lesions being exclusively located in the plantar interdigital cleft and having a more ulcerated appearance, and "B" type lesions having a thickened, crusted appearance diffusely spread across the heel. Digital photographs were transferred to a local computer hard drive where they were labeled with the cow, foot, and date metadata for each photograph. Photographs each were presented randomly to the observer and DD lesions were subsequently scored blindly with no identification present in any image.

Routine locomotion scoring was also performed throughout the study to monitor for evidence and severity of lameness. Any animal that reached a locomotion score of 4 on the 5-point lameness scale (Sprecher et al., 1997), or any animal noted by caretakers as being lame, was promptly examined by the study personnel. If lameness was associated with DD lesions, and no other cause of lameness was observed, treatment with a topical tetracycline wrap was initiated. This wrap consisted of approximately $5 \mathrm{~g}$ of oxytetracycline hydrochloride placed over lesion, covered with $10.16 \times 10.16 \mathrm{~cm}(4 \times$ 4 inch) gauze, and wrapped with a self-adherent wrap (CoFlex, Andover Healthcare Inc., Salisbury, MA). In the current study, we categorized animals into 4 possible groups based on the observed outcome following a single treatment of a clinical DD lesion (stage 3 or 4) with a tetracycline wrap. These groups were classified as (1) a lesion that did not respond to treatment (i.e., a lesion of stage 3 or 4 did not drop below a stage 3 following treatment), (2) a lesion that responded to treatment (regression to stage 2 or less) but subsequently returned to a stage 3 or 4 lesion within the time of observation, (3) a lesion that responded to treatment (regression to stage 2 or less) and remained as a stage 1 or 2 lesion without returning to normal skin or returning to a clinical lesion, and (4) a lesion that responded to treatment and returned to normal skin. These 4 outcomes were deemed as nonresponsive to treatment, 
lesion recrudescence, lesion regression, and treatment success, respectively. A treatment failure collectively included all of the first 3 categories that did not result in a lesion reverting to normal skin.

Throughout the study, metadata regarding systemic treatment, illness and other medical interventions was collected using the farm's Dairy Comp computerized record management system (Valley Agricultural Software, Tulare, CA). These metadata were used to analyze the association of these events with an increased or decreased incidence or progression of $\mathrm{DD}$. The events that were analyzed included (1) systemic diseases such as metritis, ketosis, pneumonia, and peritonitis that could potentially lead to immunosuppression, (2) systemic administration of an antibiotic, (3) topical treatments of a DD lesion due to severe lameness, and (4) periparturient stress associated with calving. These events were compared statistically using regression and ANOVA methods assuming continuously coded outcome measures and normally distributed residuals. To evaluate the effect these events had on DD lesion development, we arbitrarily required that the pre-event score had to be recorded within $14 \mathrm{~d}$ (either side) of the event, and the outcome score of the event was evaluated as the average lesion score between d 14 and 60 following the event. The 14-d window was based on the observation that lesion scores rarely changed lesions scores naturally in less than $14 \mathrm{~d}$. Even following topical treatment, which would likely have the most rapid effect on a DD lesion, lesions typically did not change morphologic appearance for approximately $14 \mathrm{~d}$. The exception to the 14-d window was for evaluation of the dry period that was based on lesion scores at dry-off (between 55 and $80 \mathrm{~d}$ precalving) and the event outcome was the score recorded during the first $14 \mathrm{~d}$ after calving. This deviation was due to the dry pen location being distant from the tilt-chute and, therefore, the inability to evaluate lesion development during this time period. Postparturient stress was evaluated by using d 14 postcalving as the event date, and the results were the average lesion score between d 14 and 60 following the event.

Statistical analyses of associations of risk factors and DD lesion scores were analyzed utilizing a regression analysis. To examine if differences existed between groups of cows and their lowest DD lesion score and recrudescence rates following treatment, a one-way ANOVA was conducted using Stata version 12 (StataCorp LP, College Station, TX). To evaluate the differences between each of these groups, post-hoc $t$-tests were conducted assuming equal variances. The differences between lactations and the percent of $\mathrm{B}$ lesions were evaluated using a 2 -sided student's $t$-test.

\section{RESULTS}

As the study was focused on the progression and regression of natural DD lesions, 6 cows were selectively removed from the study because they had no evidence of DD lesions on any foot for an extended period of time. Animals were also selected for removal if they had DD lesions that progressed to end-stage lesions on multiple feet and no changes were noted for an extended period (no lesions for $>6 \mathrm{mo}$ ) of time as well. Thirteen cows were selectively removed before the end of the study following severe illness or severe lameness resulting from non-DD foot pathology. All animals that were removed from the study were examined and treated with a topical tetracycline wrap at the time of removal if any evidence of DD lesions were present.

Of the 61 cows enrolled in this study, the average observation length was $515 \mathrm{~d}$ with a minimum of 132 and a maximum of 978 . The cows enrolled on the study were examined on a tilt-table a total of 918 times. Each examination consisted of both rear feet being digitally photographed throughout the study with some cows having each foot observed up to 28 different times in multiple lactations. The morphologic scoring system developed during this study demonstrated that DD lesions progressed systematically with lesion scores highly correlated (either one stage greater or one stage less) to previous lesion scores $(\mathrm{r}=0.7913 ; P<0.0001)$. The majority of the feet observed $(105 / 122,86 \%)$ had evidence of DD development on at least one observation while enrolled in the study. Of those 105 feet observed with developing DD lesions, 45 developed to clinical DD lesions (stage 3 or 4) while on the study. This yielded a mean incidence rate of 4 cases for every 100 foot-months at risk. For new lesions that developed while on the trial, the average time for a lesion to progress from the first morphologic changes associated with a stage 1 lesion to a clinical stage 3 lesion was $133 \mathrm{~d}$ (range $=38-315 \mathrm{~d}$, median $=105 \mathrm{~d})$. A significant $(\mathrm{r}$ $=0.2072 ; P<0.001)$ correlation was observed between the lesion statuses of the rear legs with $78 \%$ of the rear legs having a matching lesion status (i.e., lesion or no lesion). When present, lesions on both rear legs tended to progress or regress in a similar manner. When a lesion on one leg increased or decreased by a single lesion score the opposite leg was likely to increase or decrease in the same direction by a lesion score of $0.21(P<$ 0.001). Figure 1 demonstrates this co-dependence on an example subset of cows by plotting their left rear (LR) and right rear (RR) lesion scores (y-axis: 1-4) over a period of time.

Over the course of the $3 \mathrm{yr}, 1,678$ individual foot observations were scored blindly from digital photo- 
graphs using the Iowa DD scoring system. From these observations, $75 \%$ of all foot observations were scored as having either developing lesions (stage 1 and 2) or clinical lesions (stage 3 and 4). Clinical lesions comprised only $20 \%$ of all foot observations; in effect, this meant that in $55 \%$ of all observations cows had lesions that would not classically be defined as DD. A linear regression analysis of the locomotion score versus the maximum lesion score (highest lesion score on any rear foot) failed to achieve statistical significance. Likewise the highest average locomotion score was identified in animals with no DD lesions; and an ANOVA comparing the distribution of locomotion scores for each lesion stage showed the only significant difference $(P<0.05)$ was between lesion stage 0 and lesion stage 3 animals, where the lesion stage 3 animals (classic DD lesions) had the lowest average locomotion scores of all groups. Overt lameness from DD (defined as a locomotion score of 4 or greater where no other cause of significant lameness could be identified after complete physical exam) was exclusively associated with clinical lesions (stages 3 and 4). The average time from clinical lesion development (stage 3) to lameness was $161 \mathrm{~d}$ (range $=0-330$ d). Moreover, many cows maintained clinical lesions for prolonged periods of time without developing lameness.
One cow in the study was enrolled with a stage 4 lesion and maintained that lesion for $869 \mathrm{~d}$ until the conclusion of the study without ever developing lameness as defined as a locomotion score of greater than 3 on a 5-point scale (Sprecher et al., 1997). The Iowa DD scoring system differentiates stage 1 and 2 lesions into 2 distinct types of morphologic categories. These have been combined in many of the analyses as a means to calculate an increase or decrease in lesion score; however, definite differences exist in the epidemiology of the 2 morphologies. Figure 2 shows the percentage of feet that had a B type lesion by lactation. Stages B1 and B2 are much more common in first-lactation heifers than in all other lactations combined ( $41 \%$ versus an average of $14 \% ; P<0.001)$. Additionally, Table 1 shows a summary of the types of lesions present on each individual cow as well as the length of enrollment and lactations represented during the entirety of the study.

For demonstrative purposes, several representative examples of DD lesions progressing through the various stages of development are shown in Figure 3. This figure shows the lesion stages at each of our observations with several representative photos of the different lesion stages. Cow A was enrolled in the trial at the beginning of her second lactation with an existing A1 lesion. Over
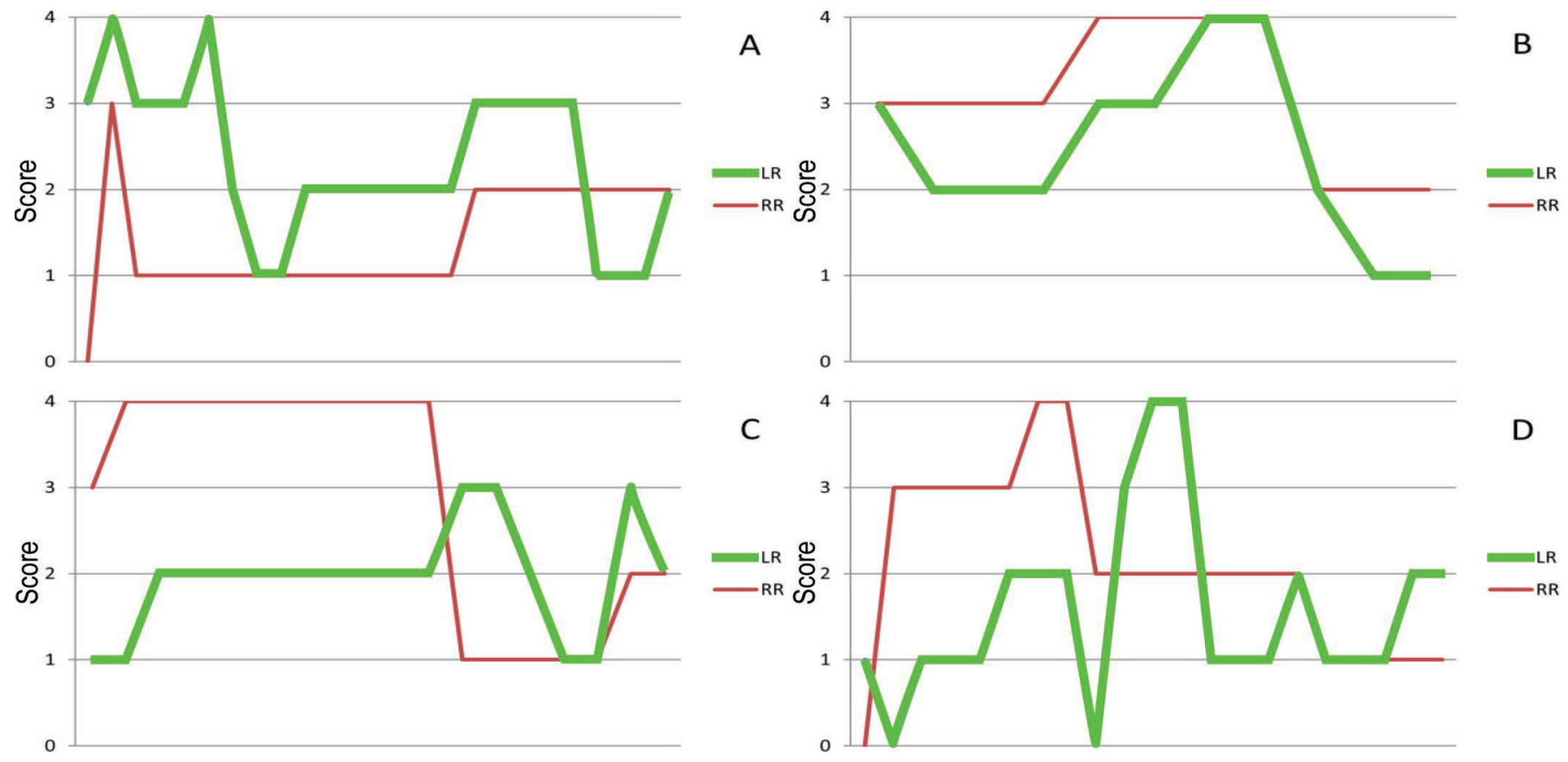

Figure 1. Individual cow rear leg morphologic lesion scores. Four representative charts (A-D) of individual cow's rear leg scores over the period of sequential observations for that cow. Chart A represents a cow that was enrolled in its second lactation and followed for $596 \mathrm{~d}$, also including the cow's third and fourth lactations. Chart B represents a cow that was enrolled in its first lactation and followed for $196 \mathrm{~d}$. Chart C represents a cow that was enrolled in its first lactation and followed for $385 \mathrm{~d}$. Chart D represents a cow that was enrolled in its first lactation and followed for $627 \mathrm{~d}$. The charts illustrate that the lesions present on both hind feet will tend to increase or decrease in the same direction of lesion severity over time. $\mathrm{LR}=$ left rear; $\mathrm{RR}=$ right rear. Color version available online. 
the first $50 \mathrm{~d}$ of the trial, the A1 lesion progressed to an A2 lesion strictly based on the size of the lesion. On the d-78 observation, an ulcerative clinical stage 3 lesion was observed, and on d 138 the hyperkeratotic characteristics of a chronic stage 4 lesion were observed. On d 272 , the lesion was treated and rapidly regressed back to a stage A2 lesion, where it remained for the remainder of the time on the trial ( $428 \mathrm{~d}$ ). Cow B was enrolled in the study at the beginning of her first lactation. She began the trial with a B1 lesion that increased in size to a B2 lesion within the first few weeks on the trial. This lesion remained a B2 lesion for over $200 \mathrm{~d}$ until a stage 3 lesion was observed on d 242. A digital photograph is shown in Figure 2 for d 251 that shows the transition from a B2 lesion to a stage 3 lesion. The criterion for a stage 3 lesion is ulceration, and a small area of ulceration was noted when the crusts of a B2 lesion were lost. This small area quickly progressed to a more obvious stage 3 lesion at the d-290 observation. On d 290, the lesion was treated and over the next several months the lesion regressed back through several of the preclinical lesion stages. This lesion is typical of many of our first-lactation heifers that originally developed a DD lesion through B lesions; following treatment, these regressed back to A type lesions.

A total of 52 topical tetracycline wraps were applied to stage 3 and 4 lesions over the course of the study, either due to lameness associated with a DD lesion or a lesion treated at the time a cow was removed from the study. As some of the feet were observed for an extended period of time, many of the feet that developed end-stage

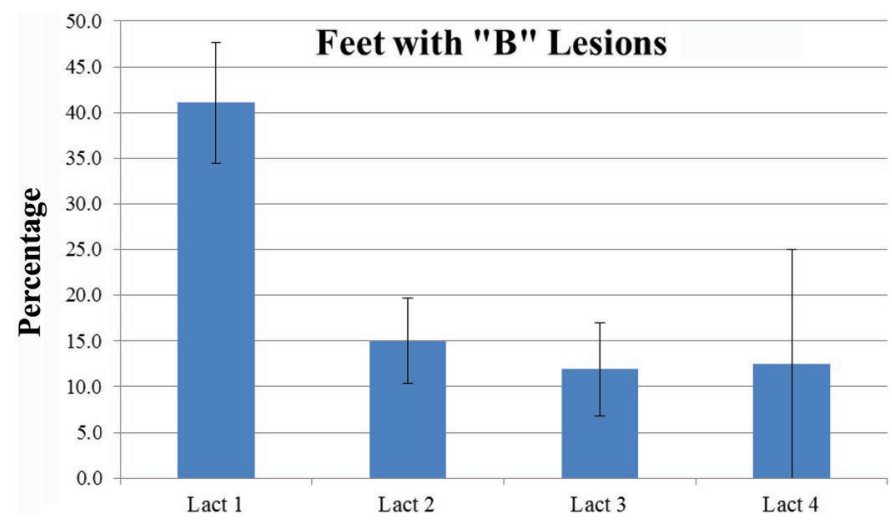

Figure 2. The percentage of the feet that had a B type lesion by lactation (Lact). The percentage is based on the number of feet that had B lesion in cows undergoing lesion development. Stages B1 and B2 are statistically more common in first-lactation heifers compared to all other lactations (41 vs. an average of $14 \%, P<0.001$ ). B-type lesions differ from A-type lesions in that they develop more diffusely across the heel and have a thickened, crusted appearance, whereas A-type lesions initiate on the plantar interdigital cleft and have a more ulcerated appearance. Color version available online. lesions were treated and redeveloped lesions at a later time. These treated lesions were monitored and subsequently scored during routine tilt-table observations. Of the 52 lesions treated with topical tetracycline, 43 of them were monitored for a minimum of $50 \mathrm{~d}$ to evaluate the effect of treatment and lesion recrudescence. Of these 43 lesions, 40 of them regressed to a lower lesion stage following treatment. Table 2 shows a summary of the subsequent observations and the lowest lesion stage reached following treatment. Despite the fact that topical tetracycline was effective in reducing lesion scores, it was highly ineffective (9\%) at returning DD lesions back to normal skin. Significant differences $(P<0.05)$ were observed between the lowest lesion score observed following treatment and the rate of recrudescence of disease. Lesions that regressed to stage 2 following treatment had the highest rate of recrudescence (59\%) and the lowest number of days (120) for these lesions to return to clinical lesions (stage 3 or 4 ). The lesions that regressed to stage 1 following treatment were less likely $(P<0.05)$ to return $(29 \%)$, and the days required for these lesions to return was longer (224) than those that only regressed to stage 2 . Of the 4 lesions that completely healed (stage 0), none of them developed clinical lesions again during their observation period, which averaged $420 \mathrm{~d}$ following treatment for these 4 animals. Of these 4 lesions, 2 of them had follow-up biopsies (69 and $90 \mathrm{~d}$ post-treatment) after regressing to a completely healed state (stage 0 ). Both of these were submitted for histopathology and were classified as normal tissue with no signs of infectious agents. None of these 4 lesions were included in the subset of stage 0 lesions included in the metagenomics analysis. There was no statistical difference $(P=0.12)$ between recrudescence rates between those that regressed to stage 0 versus stage 1 , but a highly significant difference $(P<0.01)$ was observed in recrudescence rates between those that regressed to stage 0 versus stage 2 . We found no difference $(P=0.11)$ between the recrudescence rates of lesion classified as stage 3 or stage 4 at the time of treatment.

Over the $3 \mathrm{yr}$ of the study, a total of 369 events were recorded in the metadata categories listed in Table 3. Of the 369 events, 177 had lesion scores recorded during both the pre-event window and the window for evaluation following the event. As many events occurred simultaneously with other events (e.g., systemic disease often occurred along with systemic antibiotic therapy), a separate analysis is shown in Table 3 (third column) that lists events that occurred independently of anything else for that animal. Two events were considered simultaneous if they occurred within $14 \mathrm{~d}$ of each other for the same reasons discussed above. The elimination 
Table 1. Lesion summary data for all cows in the study

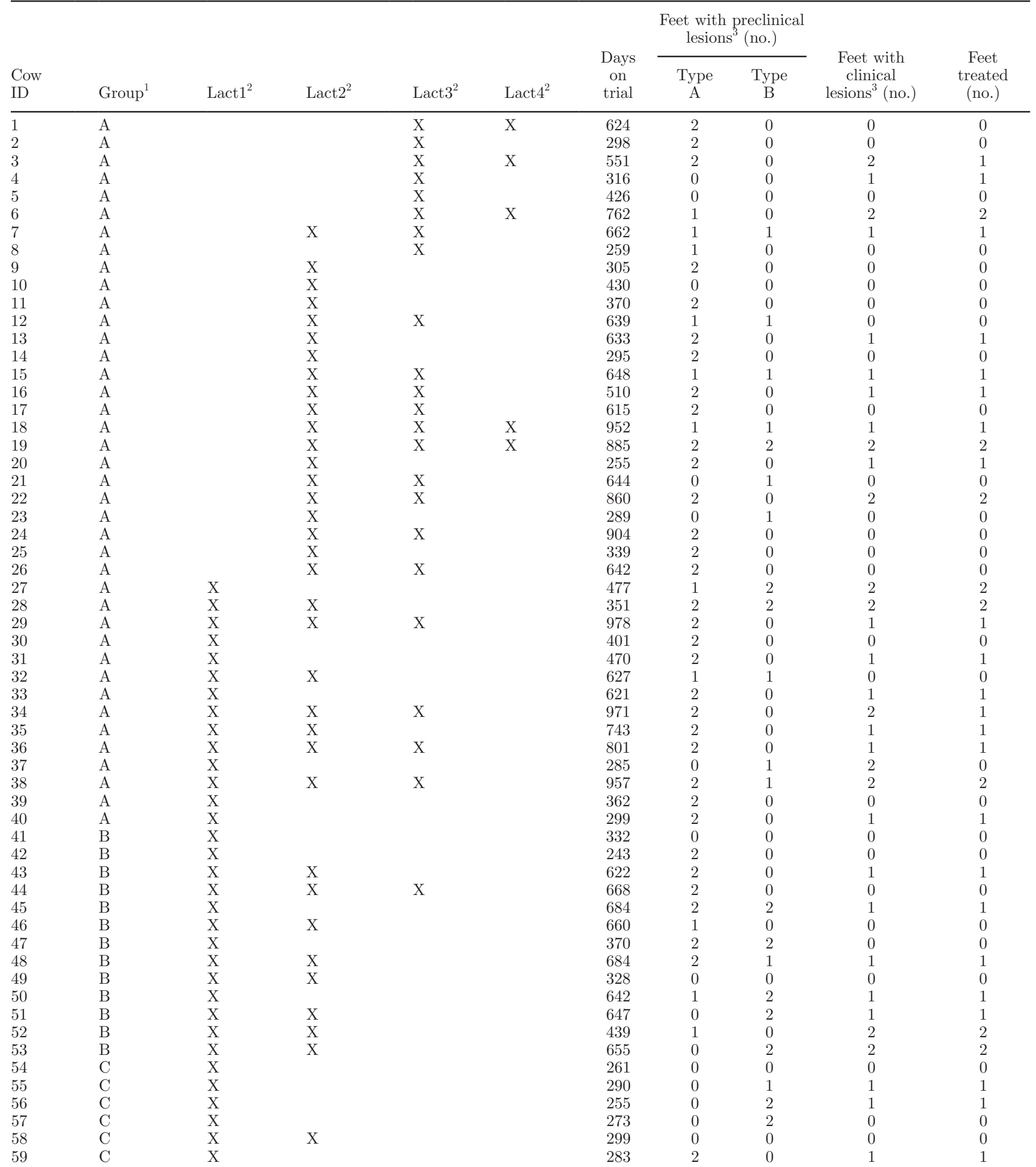


Table 1 (Continued). Lesion summary data for all cows in the study

\begin{tabular}{|c|c|c|c|c|c|c|c|c|c|c|}
\hline \multirow[b]{2}{*}{$\begin{array}{l}\text { Cow } \\
\text { ID }\end{array}$} & \multirow[b]{2}{*}{ Group $^{1}$} & \multirow[b]{2}{*}{ Lact $^{2}$} & \multirow[b]{2}{*}{ Lact $^{2}$} & \multirow[b]{2}{*}{ Lact $^{2}$} & \multirow[b]{2}{*}{ Lact $4^{2}$} & \multirow[b]{2}{*}{$\begin{array}{l}\text { Days } \\
\text { on } \\
\text { trial }\end{array}$} & \multicolumn{2}{|c|}{$\begin{array}{l}\text { Feet with preclinical } \\
\text { lesions }^{3} \text { (no.) }\end{array}$} & \multirow[b]{2}{*}{$\begin{array}{c}\text { Feet with } \\
\text { clinical }^{3} \\
\text { lesions }{ }^{3} \text { (no.) }\end{array}$} & \multirow[b]{2}{*}{$\begin{array}{l}\text { Feet } \\
\text { treated } \\
\text { (no.) }\end{array}$} \\
\hline & & & & & & & $\begin{array}{c}\text { Type } \\
\text { A }\end{array}$ & $\begin{array}{c}\text { Type } \\
\text { B }\end{array}$ & & \\
\hline 60 & $\mathrm{C}$ & $\mathrm{X}$ & $\mathrm{X}$ & & & 132 & 0 & 1 & 0 & 0 \\
\hline 61 & $\mathrm{C}$ & $\mathrm{X}$ & $\mathrm{X}$ & & & 247 & 2 & 0 & 2 & 1 \\
\hline
\end{tabular}

${ }^{1}$ Group A consisted of first-, second-, and third-lactation animals that were enrolled as they calved in 2010. They were not evaluated for digital dermatidis (DD) status prior to enrollment and no history of DD lesions was available at the time of enrollment. Group B was enrolled in 2011 and consisted of first-lactation animals that were screened prior to enrollment for the presence of DD lesions and any animal with evidence of classic end-stage DD lesions was excluded from the study. Group C was enrolled in 2012 and consisted only of first-lactation animals that were screened prior to enrollment for the presence of DD lesions and any animal with evidence of either developing or clinical DD lesions was excluded from the study.

${ }^{2}$ Each lactation (Lact) checked indicates that foot observations were made during that lactation.

${ }^{3}$ The number of preclinical (stages 1 and 2) and clinical (stages 3 and 4 ) lesions indicates the number of rear feet that developed to that stage at some point while on study.

of simultaneous events lowered the number of events that qualified for analysis to 121 . Of the 5 types of events recorded, the only event that significantly altered the development of DD lesions was topical tetracycline therapy, which decreased lesion scores by more than one score on average. When compared with systemic antibiotics, topical tetracycline had a significantly better effect $(P<0.001)$ on decreasing DD lesion scores.

\section{DISCUSSION}

This study clearly demonstrates that the Iowa DD scoring system used for the analysis of DD lesion development serves as an effective method to study lesion progression and regression. It has been previously shown that DD lesions differ morphologically over time, and several scoring systems have been devised (Döpfer et al., 1997) in an attempt to categorize the progression of lesion development. We believe that the Iowa DD scoring system has several significant advantages over previously described scoring systems. First, it allows for a more detailed evaluation of the development of early-stage lesions by providing 4 distinct classifications instead of incorporating all developing lesions into one category. Second, we demonstrate in this manuscript that the lesion score following therapy provides useful prognostic data regarding the likelihood of recrudescence and the period of time until recrudescence. We are not aware of any other scoring system that has been demonstrated to provide this prognostic information. Finally, the Iowa DD scoring system is the only scoring system that has also been statistically validated to have a unique microbiota associated with each of the specific morphologic lesions (Krull et al., 2014). Based on these observations we believe that the Iowa DD scoring system could also be a useful tool for herd-level surveillance during routine trimming and foot care. In comparison to other scoring systems, the greater emphasis placed on classifying the earliest lesion stages and their associated prognostic outcomes provides for an evidence based approach to managing DD prevention measures on farms. The earlier recognition of lesions provided by the scoring system allows for earlier intervention and prognostication, which in turn allows for better decision making regarding the necessity for retreatment of lesions.

The relationship between DD and interdigital dermatitis (ID) has been debated in the literature. In our study we defined ulcerative lesions exclusively located in the plantar skin adjacent to the interdigital cleft as type A DD lesions. It was observed that many additional cows had ulcerative lesions within the interdigital space or the deep pocket of the interdigital cleft that did not extend into the plantar aspect of the interdigital cleft. Those lesions were considered ID and were not scored with the Iowa DD scoring system. Conversely, lesions were observed in which ulceration began within the interdigital space and subsequently spread to the plantar skin adjacent to the interdigital cleft. In those instances, lesions were designated as A1 DD lesions. It was our observation that cows could have ID without DD, DD without ID, or a combination of ID and DD. What degree of overlap exists in the microbial populations of each of these distinct lesions types is not presently known due to a lack of metagenomic data derived from ID cases; however, it is likely that some overlap in microbial populations will exist due to proximity and similarity of bacterial niche. Based on the results of the present study, 2 key points regarding the epidemiology of DD can be made. First, all advanced DD lesions (stage 3 or 4 ) are preceded by an early stage 1 and 2 DD lesion. Therefore, the identification of one of these 
early lesion types in the plantar skin adjacent to the interdigital cleft should alert the clinician or trimmer to the predisposition for that lesion to progress to an advanced DD lesion in herds with endemic DD. Second, not all early lesions progress to advanced lesions. Some remained morphologically similar to early lesions for a prolonged period and some resolve without treatment. The ability for an early lesion to progress to an advanced lesion is believed to be influenced by a variety of factors, including (1) exposure to and colonization of
A

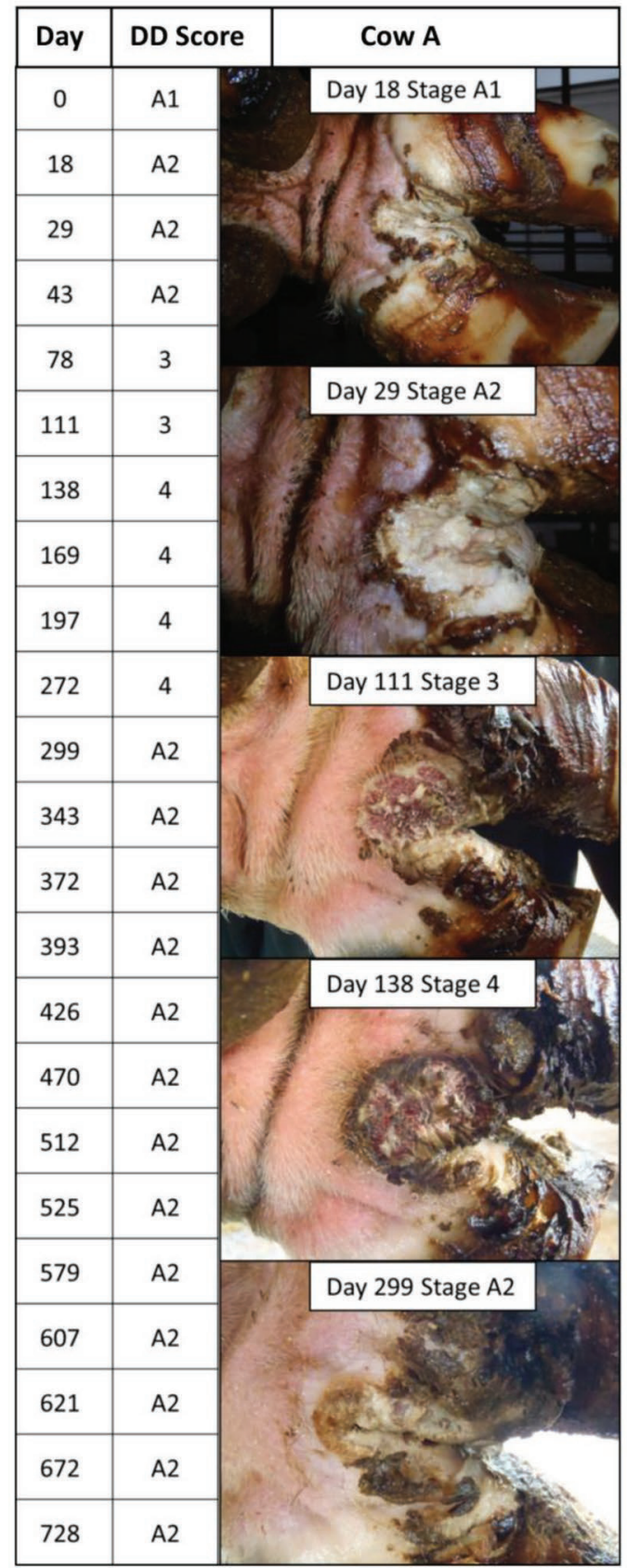

B

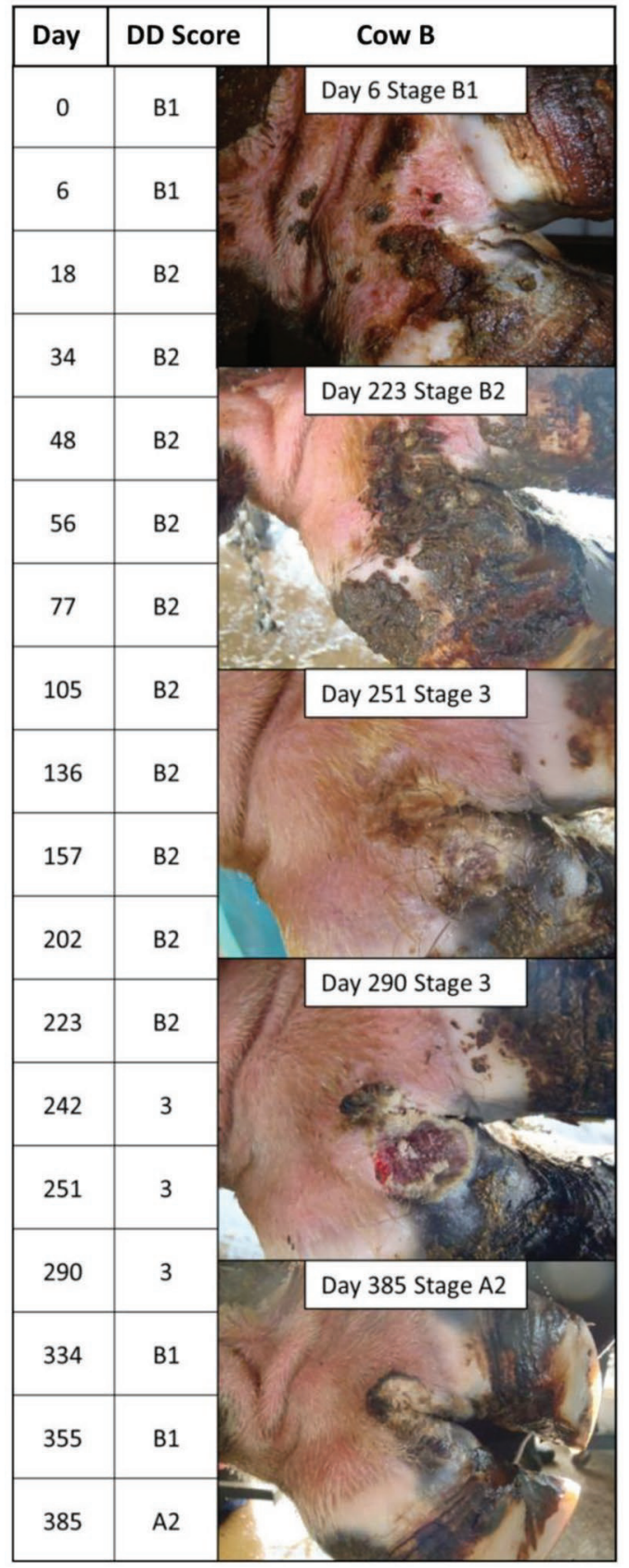

Figure 3. A list of lesion scores over a period of time are listed for 2 different cow rear feet. A subset of lesion morphologies are shown that are most representative of each digital dermatitis (DD) lesion score. Both of these feet progressed systematically through the Iowa DD scoring system, with cow A progressing with A-type lesions and cow B progressing with B-type lesions. Both feet were treated during their observation period with cow A being treated on d 272 and cow B being treated on d 290. See text for additional details. Color version available online. 
Table 2. Digital dermatitis (DD) lesion treatment and recrudescence

\begin{tabular}{lccccc}
\hline $\begin{array}{l}\text { Lowest DD } \\
\text { score }^{1}\end{array}$ & $\begin{array}{c}\text { Lesions } \\
\text { (no.) }\end{array}$ & $\begin{array}{c}\text { Recur }^{2} \\
(\text { no. })\end{array}$ & $\begin{array}{c}\text { Prevalence } \\
(\%)\end{array}$ & Days to recur $^{3}$ & Days monitored $^{4}$ \\
\hline 3 & 3 & 3 & 100 & 0 & $298(265-323)$ \\
2 & 22 & 13 & $59^{\mathrm{ab}}$ & $120(85-431)$ & $289(52-603)$ \\
1 & 14 & 4 & $29^{\mathrm{a}}$ & $224(52-306)$ & $265(56-679)$ \\
0 & 4 & 0 & $0^{\mathrm{b}}$ & NA $^{5}$ & $420(252-567)$ \\
\hline
\end{tabular}

a,b Superscripts of the same letter indicate statistical significance between recurrence rates $(P<0.05)$.

${ }^{1}$ The lowest DD score refers to the lowest DD lesion score that lesions regressed to following a single treatment with topical tetracycline. All DD lesions treated in this analysis were initially classified as either stage 3 or 4 clinical lesions at the time of treatment. The DD lesion scores were based on the Iowa DD Lesion Scoring System.

${ }^{2}$ Recur refers to the number of lesions that return to a DD lesion score of 3 or 4 during the time period observed.

${ }^{3}$ Days to recur indicates the time from treatment to the time in which the lesions returned to a stage 3 or 4 . In cases in which lesions did not regress any lower than stage 3 , these would be considered treatment failures and thus do not have a time to recur.

${ }^{4}$ The amount of time each lesion was observed is shown in the last column, and also shown is the mean time observed following treatment and the range of time for individual lesions.

${ }^{5}$ Not applicable.

the early lesion by bacterial agents necessary for this transition (i.e., the bacteria that differentiate advanced lesions from early lesions based on our prior work, described in Krull et al., 2014), (2) host genetics and immunity, and (3) environmental conditions. Clinical experience would also suggest that in a small number of dairy herds the only types of DD lesions observed are early lesions (stage 1 and 2). At present, it is unclear how the factors described in the previous sentence work together to limit the transition to advanced lesions, and additional research to answer this interesting question is warranted.

Several other important observations were noted over the course of the 3 yr that have implications as to how we view the incidence of DD. The fact that $75 \%$ of observations had lesion scores greater than zero suggests that when cows are not exposed to any DD prevention measures, the majority of feet in farms with endemic DD could develop some level of DD. With only $26 \%$ of those lesions being clinical lesions that would be recog-

Table 3. The effect of various events of digital dermatitis (DD) lesion stage

\begin{tabular}{|c|c|c|c|c|c|c|}
\hline \multirow[b]{2}{*}{ Event } & \multicolumn{2}{|c|}{$\begin{array}{l}\text { Simultaneous } \\
\text { events }\end{array}$} & \multicolumn{2}{|c|}{$\begin{array}{l}\text { Independent } \\
\text { events }\end{array}$} & \multirow[b]{2}{*}{ Criteria for event score } & \multirow[b]{2}{*}{ Criteria for event result } \\
\hline & No. & $\begin{array}{l}\text { DD score } \\
\text { change }\end{array}$ & No. & $\begin{array}{l}\text { DD score } \\
\text { change }\end{array}$ & & \\
\hline Systemic disease $^{1}$ & 42 & -0.01 & 19 & 0.13 & \pm 14 d of event ${ }^{2}$ & +14 to $60 \mathrm{~d}$ after event ${ }^{3}$ \\
\hline Systemic antibiotics ${ }^{4}$ & 26 & -0.24 & 7 & -0.07 & $\pm 14 \mathrm{~d}$ of event ${ }^{2}$ & +14 to $60 \mathrm{~d}$ after event ${ }^{3}$ \\
\hline Topical DD therapy $^{5}$ & 27 & -1.08 & 21 & -1.17 & $\pm 14 \mathrm{~d}$ of event ${ }^{2}$ & +14 to $60 \mathrm{~d}$ after event ${ }^{3}$ \\
\hline
\end{tabular}

${ }^{1}$ Systemic disease included coliform mastitis, ketosis, metritis, pneumonia, left displaced abomasum, and peritonitis.

${ }^{2}$ Due to some events occurring more than $14 \mathrm{~d}$ from an examination, those events were excluded from analysis. The event result for these events was the average lesion score from observations made between 14 and $60 \mathrm{~d}$ after event.

${ }^{3}$ The effect of dry period was slightly modified as cows were not available for observations during the dry period. The event score for this analysis was the final examination score prior to dry-off that was less than $80 \mathrm{~d}$ from calving.

${ }^{4}$ The systemic antibiotics were not given to treat digital dermatitis, but the effect on DD lesions was measured when systemic antibiotics were given to treat a number of other conditions.

${ }^{5}$ Topical DD therapy was a single topical wrap with tetracycline. For these events, the DD lesion score at the time of event was the average lesion score when measured within $14 \mathrm{~d}$ of an event.

${ }^{6}$ The event result for the dry period was the first lesion score following calving that was less than $14 \mathrm{~d}$ from calving.

${ }^{7}$ The effects of periparturient period were evaluated by using d 14 following calving as the event and the result was measured 14 to $60 \mathrm{~d}$ following event.

${ }^{8}$ The column indicating independent event outcome included only events in which no other events happened within $14 \mathrm{~d}$ of the event. The simultaneous event column included these events. 
nized by hoof trimmers and practitioners as classic DD lesions, estimates of incidence rates and prevalence are likely greatly underestimated in the literature. We did not directly measure prevalence, as this was an entryrestricted cohort study and not a single time point analysis of prevalence across all animals in the herd. To compare our data to the other literature on prevalence, we used our incidence rate of 4.36 DD lesions per 100 feet-months and an average DD lesion lasting 5.26 mo in our study to calculate a prevalence of $22.9 \%$ (Indrayan, 2012). Although we did not directly measure prevalence, our calculation of prevalence was in line with other studies estimates of 20 to $26 \%$ in freestall barns (Cramer et al., 2008). Importantly, the use of lameness in herds as a proxy for the prevalence of $\mathrm{DD}$ would underestimate the number of cows affected with $\mathrm{DD}$, as we observed that the majority of clinical lesions fail to induce lameness. The use of locomotion score as a way to identify cows with DD was shown to be very inconsistent, with many cows maintaining clinical lesions for months to years without ever reaching a stage 4 or 5 locomotion score. This is consistent with data from Frankena et al. (2009), in which only 39\% of cows with severe DD lesions ever showed any of lameness.

The finding of $\mathrm{B}$ lesions being more frequently observed in first-lactation animals is interesting. Given their age and the relatively low incidence of DD in heifers on this farm, this finding might suggest that DD naïve animals are more likely to develop this subtype of lesion during the first disease exposure. Likewise, the A lesions may represent a natural progression of lesions in animals with prior exposure. As some of the cows enrolled were already in their second and third lactation and their history of DD lesions was unknown upon enrollment, we were unable to determine if the lesions in these cows were proceeded by other lesions that had healed at the time of enrollment. Therefore, additional work regarding the difference in the natural history and epidemiology between type A and B lesions is warranted and may provide a better understanding of the initiation and host response to this disease process.

Figure 4 is a graphical working hypothesis of lesion development and regression over time representative of what was commonly observed. Digital dermatitis lesions progress from normal skin through either A or $\mathrm{B}$ lesions to the final end-stage lesions (stages 3 and 4). A full description of the Iowa DD scoring system is provided by Krull et al. (2014), with A2 and B2 lesions being larger in size than A1 and B1 lesions. All clinical lesions observed in this study progressed through these stages before becoming stage 3 lesions. Likewise, the ulcerative and hyperemic lesions, characteristic of stage 3 lesions, always preceded the more chronic stage 4 lesions that are proliferative and hyperkeratotic. Following treatment of stage 3 or 4 lesions, a period of time (usually less than $10 \mathrm{~d}$ from a single point treatment)

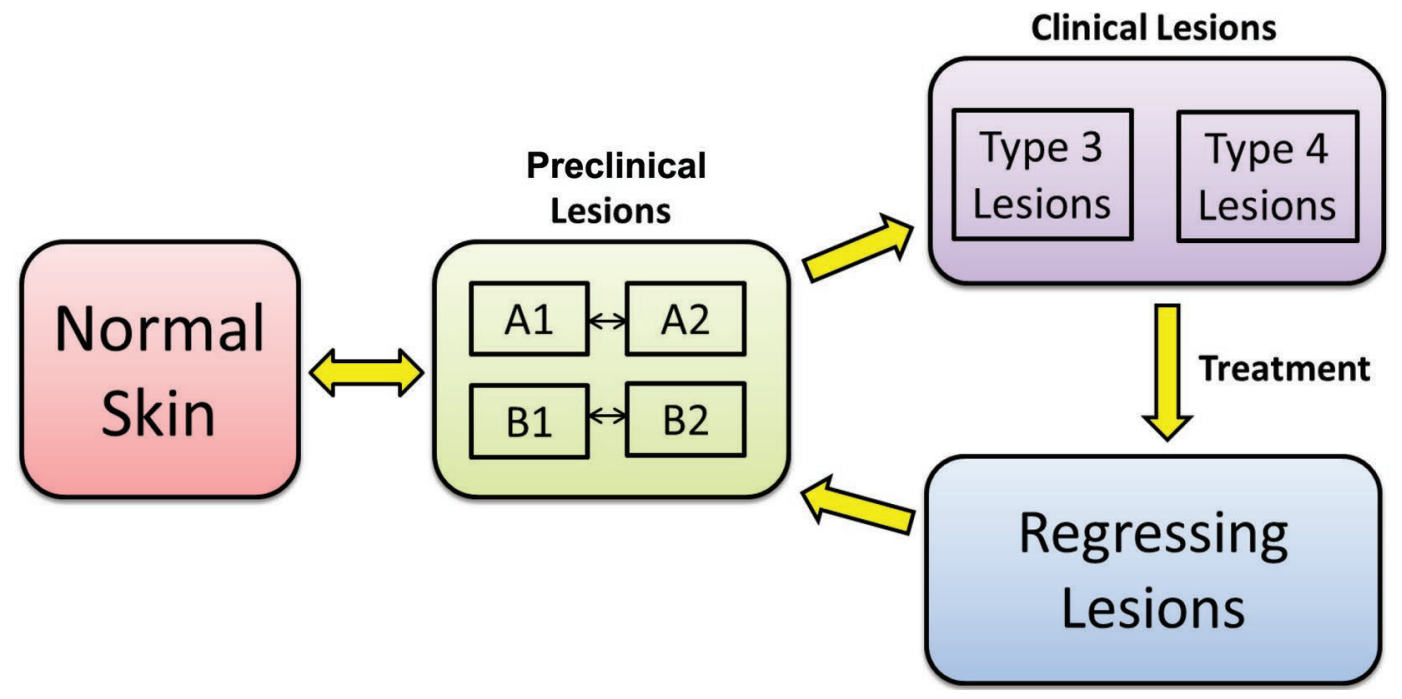

Figure 4. Digital dermatitis (DD) lesion development model. The DD lesions always begin with a change from normal skin to either A1 or B1 lesions. These lesions can remain in this preclinical lesion stage for an indefinite period of time transitioning between the various lesion categories. At some point, an area of these preclinical lesions begins to ulcerate and become hyperemic indicative of a stage 3 lesion. If left untreated, these stage 3 lesions will eventually hypertrophy and exhibit the classic "hairy heel wart" appearance. Following treatment of the active or clinical lesions, there is a period of time in which much of the tissue becomes necrotic and eventually sloughs. During this time, it is difficult to accurately stage the DD lesion. Once the necrotic tissue is gone, unless there is a treatment failure, the lesions can again be classified in the preclinical lesion category. Color version available online. 
occurred in which the outside layer of the epidermis became black and necrotic. Until this necrotic tissue sloughs, it is difficult to determine the amount of lesion regression and which lesion stage the treated lesion will become. As shown in Table 2, rarely does a singlepoint treatment yield the result of a lesion returning to normal skin (stage 0). The majority of time the lesion will regress to the preclinical category (stage 1 or 2). As demonstrated in our study, the amount of lesion regression is highly correlated with the likelihood of recrudescence and the time to recrudescence.

The natural history and epidemiology of DD lesion development observed in our study yielded similar results to Relun et al. (2013) in a long-term follow-up of over 4,000 cows from French dairy farms. That study found the median time for the occurrence of a new DD lesion to be $146 \mathrm{~d}$, whereas our average time for a lesion to develop was $133 \mathrm{~d}$. The incidence rate from both studies was 4 new cases per 100 cow foot-months. The increased risk for an animal to have a lesion on the contralateral foot when a lesion was observed on a single rear foot in the present study was consistent with the findings of the French study. The major difference is that our study was conducted on a single farm in the United States with a single breed (Holstein) whereas Relun et al. (2013) was done across multiple farms in France with multiple breeds and management systems very different from the US production system. As in our study, they conducted all examinations on a tilt-table instead of using parlor evaluations. The fact that the results were very similar despite the location and study population differences suggests that DD development is likely to be consistent world-wide and across breeds.

The high rate of lesion recrudescence following a single topical treatment has been repeatedly shown in multiple studies (Holzhauer et al., 2011; Berry et al., 2012; Cutler et al., 2013). The majority of papers reporting the treatment success and recrudescence only followed treated lesions for $30 \mathrm{~d}$ or less. Only the study by Berry et al. (2012) followed any of the treatments past $30 \mathrm{~d}$ to evaluate treatment success when re-examined at $341 \mathrm{~d}$. The results of this present study indicate a high rate of DD lesion recrudescence following a single tetracycline treatment (44\%) when followed for an extended period of time (as long as $679 \mathrm{~d}$ ). The average time required for these lesions to recrudesce was also much longer than most of the studies had reported as follow-up time after treatment. Additionally, none of 17 lesions that initially responded to treatment and later redeveloped DD lesions did so in less than $50 \mathrm{~d}$. This calls into question the outcomes of treatment studies that assess lesion recrudescence at $30 \mathrm{~d}$, as is commonly reported in the literature. This short 30-d follow-up period makes it difficult to determine if the lesions are treatment failures or lesion recrudescence. Our data showed that a single topical treatment of tetracycline had a treatment success rate of $9 \%$, which is lower than previous reports. This discrepancy is partly due to the definition of treatment success, which in our study requires the skin returning to normal. In studies that assessed complete healing of the lesion to normal skin, success rates ranged from 14 to 39\% (Shearer and Hernandez, 2000, Nishikawa and Taguchi, 2008), whereas studies that defined treatment success as a decreased lesion size or decreased pain the success ranged from 47 to 73\% (Berry et al., 2010; Cutler et al., 2013). A large discrepancy also exists in the duration of follow-up between these studies, which may have also contributed to the differences. Importantly, we were able to use the Iowa DD scoring system to evaluate lesion morphology following treatment and also have validated its use as a prognostic tool for lesion recrudescence.

The collection of metadata during the course of the study was done in an attempt to identify the effect of various management factors and interventions on DD lesion development. We recorded every event that happened to the cows on trial, including mastitis, ketosis, displaced abomasum, metritis, pneumonia, and so on, that occurred over these 3 yr. As we examined our data, too few instances were noted of each of these events separately to derive any conclusions about their effects on DD lesions. Therefore, we combined all of these events to determine if systemic illness of any kind had any effect on lesion development. As we were also looking at the effects of systemic antibiotics and the periparturient period, it was difficult to assess many of these things independently of each other. Many times a case of metritis was obviously associated with the periparturient period as well as systemic antibiotics used to treat the condition. The data shown in Table 3 summarizes these effects with and without these confounders included. No effect was observed on lesion score from events that would be expected to be associated with immunosuppression, including systemic disease, dry period, or periparturient period; although this should be interpreted with caution, as this was not the primary focus of the study.

Although no antibiotics are labeled by regulatory agencies to treat DD in cattle in the United States, we were interested to see if antibiotics given for different disease would affect DD lesion development. Although systemic tetracycline has been shown to have limited success (Loureiro et al., 2010), systemic cefquinome (not an approved product in the United States) has been shown to reduce DD lesion size in the United Kingdom (Laven, 2006). In the few cases of the present study 
where systemic antibiotics were given for nonsystemic disease (typically footrot cases), systemic antibiotics did not have a significant $(P>0.05)$ effect on lesion score. For all of the events measured, the only event that was statistically significant $(P<0.05)$ was topical tetracycline therapy. This was not surprising considering the many papers documenting treatment success using tetracycline (Nishikawa and Taguchi, 2008; Berry et al., 2010; Loureiro et al., 2010; Cutler et al., 2013); however, the fact that the Iowa DD scoring system was able to be used to document this in a statistical manner was encouraging for its use in future treatment trials.

The observations from our study provide important insights into how DD lesions progress from normal skin to the readily identifiable clinical disease. The Iowa DD lesion scoring system was shown to be a valuable tool in describing these changes, as well as serving as a way to measure the outcome of DD intervention strategies. Future research into whether more aggressive treatment as a means to return DD lesions back to normal skin would be beneficial in lowering the recrudescence rate is warranted. Additionally, further studies evaluating the economics of treating the more prevalent preclinical lesions before developing into clinical DD lesions should be looked at to reduce lameness and production losses associated with clinical lesions.

\section{ACKNOWLEDGMENTS}

The authors thank Cory Haglund, Michael Slattery, Tim Kruse, Lance Dahlquist, Mackenzie Dickson, and the Iowa State University Dairy Farm staff for their assistance in sample collection and animal management. Funds for this research project were provided by Boehringer Ingelheim Vetmedica Inc. (St. Joseph, MO) and Iowa State University startup funds.

\section{REFERENCES}

Becker, J., A. Steiner, S. Kohler, A. Koller-Bahler, M. Wuthrich, and M. Reist. 2014. Lameness and foot lesions in Swiss dairy cows: I. Prevalence. Schweiz. Arch. Tierheilkd. 156:71-78.

Berry, S. L., D. H. Read, T. R. Famula, A. Mongini, and D. Doepfer 2012. Long-term observations on the dynamics of bovine digital dermatitis lesions on a California dairy after topical treatment with lincomycin HCl. Vet. J. 193:654-658.

Berry, S. L., D. H. Read, R. L. Walker, and T. R. Famula. 2010. Clinical, histologic, and bacteriologic findings in dairy cows with digital dermatitis (footwarts) one month after topical treatment with lincomycin hydrochloride or oxytetracycline hydrochloride. J. Am. Vet. Med. Assoc. 237:555-560.

Blowey, R. W., and M. W. Sharp. 1988. Digital dermatitis in dairy cattle. Vet. Rec. 122:505-508.

Britt, J. S., M. C. Carson, J. D. von Bredow, and R. J. Condon. 1999. Antibiotic residues in milk samples obtained from cows after treatment for papillomatous digital dermatitis. J. Am. Vet. Med. Assoc. 215:833-836.
Brown, C. C., P. D. Kilgo, and K. L. Jacobsen. 2000. Prevalence of papillomatous digital dermatitis among culled adult cattle in the southeastern United States. Am. J. Vet. Res. 61:928-930.

Bruijnis, M. R., H. Hogeveen, and E. N. Stassen. 2010. Assessing economic consequences of foot disorders in dairy cattle using a dynamic stochastic simulation model. J. Dairy Sci. 93:2419-2432.

Capion, N., S. M. Thamsborg, and C. Enevoldsen. 2008. Prevalence of foot lesions in Danish Holstein cows. Vet. Rec. 163:80-85.

Capion, N., S. M. Thamsborg, and C. Enevoldsen. 2009. Prevalence and severity of foot lesions in Danish Holstein heifers through first lactation. Vet. J. 182:50-58.

Cha, E., J. A. Hertl, D. Bar, and Y. T. Grohn. 2010. The cost of different types of lameness in dairy cows calculated by dynamic programming. Prev. Vet. Med. 97:1-8.

Cramer, G., K. D. Lissemore, C. L. Guard, K. E. Leslie, and D. F. Kelton. 2008. Herd- and cow-level prevalence of foot lesions in Ontario dairy cattle. J. Dairy Sci. 91:3888-3895.

Cutler, J. H., G. Cramer, J. J. Walter, S. T. Millman, and D. F. Kelton. 2013. Randomized clinical trial of tetracycline hydrochloride bandage and paste treatments for resolution of lesions and pain associated with digital dermatitis in dairy cattle. J. Dairy Sci. 96:7550-7557.

Döpfer, D., A. A. H. M. ter Huurne, J. L. Cornelisse, A. J. A. M. van Asten, A. Koopmans, F. A. Meijer, Y. H. Schukken, I. Szakall, W. Klee, and R. B. Bosma. 1997. Histological and bacteriological evaluation of digital dermatitis in cattle, with special reference to spirochaetes and Campylobacter faecalis. Vet. Rec. 140:620-623.

Fabian, J., R. A. Laven, and H. R. Whay. 2014. The prevalence of lameness on New Zealand dairy farms: A comparison of farmer estimate and locomotion scoring. Vet. J. 201:31-38.

Frankena, K., J. G. Somers, W. G. Schouten, J. V. van Stek, J. H. Metz, E. N. Stassen, and E. A. Graat. 2009. The effect of digital lesions and floor type on locomotion score in Dutch dairy cows. Prev. Vet. Med. 88:150-157.

Hernandez, J., and J. K. Shearer. 2000. Efficacy of oxytetracycline for treatment of papillomatous digital dermatitis lesions on various anatomic locations in dairy cows. J. Am. Vet. Med. Assoc. 216:1288-1290.

Holzhauer, M., C. J. Bartels, M. van Barneveld, C. Vulders, and T. Lam. 2011. Curative effect of topical treatment of digital dermatitis with a gel containing activated copper and zinc chelate. Vet. Rec. 169:555.

Holzhauer, M., C. Hardenberg, C. J. Bartels, and K. Frankena. 2006 Herd- and cow-level prevalence of digital dermatitis in the Netherlands and associated risk factors. J. Dairy Sci. 89:580-588.

Hulek, M., I. Sommerfeld-Stur, and J. Kofler. 2010. Prevalence of digital dermatitis in first lactation cows assessed at breeding cattle auctions. Vet. J. 183:161-165.

Indrayan, A. 2012. Medical Biostatistics. 3rd ed. CRC Press, New York, NY.

Knappe-Poindecker, M., M. Gilhuus, T. K. Jensen, K. Klitgaard, R. B. Larssen, and T. Fjeldaas. 2013. Interdigital dermatitis, heel horn erosion, and digital dermatitis in 14 Norwegian dairy herds. J. Dairy Sci. 96:7617-7629.

Krull, A. C., J. K. Shearer, P. J. Gorden, V. L. Cooper, G. J. Phillips, and P. J. Plummer. 2014. Deep sequencing analysis reveals temporal microbiota changes associated with development of bovine digital dermatitis. Infect. Immun. 82:3359-3373.

Laven, R. A. 2006. Efficacy of systemic cefquinome and erythromycin against digital dermatitis in cattle. Vet. Rec. 159:19-20.

Laven, R. A., and H. Hunt. 2001. Comparison of valnemulin and lincomycin in the treatment of digital dermatitis by individually applied topical spray. Vet. Rec. 149:302-303.

Loureiro, M. G., C. A. Rodrigues, E. S. Nascimento, C. Esteban, S. H. Perri, and C. R. Anhesini. 2010. Efficacy of topical and systemic treatments with oxytetracycline for papillomatous digital dermatitis in cows. Arquivo Brasileiro Med. Veterinaria Zootecnia 62:13-22.

Manske, T., J. Hultgren, and C. Bergsten. 2002. Topical treatment of digital dermatitis associated with severe heel-horn erosion in a Swedish dairy herd. Prev. Vet. Med. 53:215-231. 
Moore, D. A., S. L. Berry, M. L. Truscott, and V. Koziy. 2001. Efficacy of a nonantimicrobial cream administered topically for treatment of digital dermatitis in dairy cattle. J. Am. Vet. Med. Assoc. 219:1435-1438.

Nishikawa, A., and K. Taguchi. 2008. Healing of digital dermatitis after a single treatment with topical oxytetracycline in 89 dairy cows. Vet. Rec. 163:574-576.

Read, D. H., and R. L. Walker. 1998. Papillomatous digital dermatitis (footwarts) in California dairy cattle: Clinical and gross pathologic findings. J. Vet. Diagn. Invest. 10:67-76.

Relun, A., A. Lehebel, M. Bruggink, N. Bareille, and R. Guatteo. 2013. Estimation of the relative impact of treatment and herd management practices on prevention of digital dermatitis in French dairy herds. Prev. Vet. Med. 110:558-562.

Shearer, J. K., and J. Hernandez. 2000. Efficacy of two modified nonantibiotic formulations (Victory) for treatment of papillomatous digital dermatitis in dairy cows. J. Dairy Sci. 83:741-745.
Sprecher, D. J., D. E. Hostetler, and J. B. Kaneene. 1997. A lameness scoring system that uses posture and gait to predict dairy cattle reproductive performance. Theriogenology 47:1179-1187.

Sullivan, L. E., S. D. Carter, R. Blowey, J. S. Duncan, D. GroveWhite, and N. J. Evans. 2013. Digital dermatitis in beef cattle. Vet. Rec. 173:582.

USDA. (2009). Dairy 2007, Part IV: Reference of Dairy Cattle Health and Management Practices in the United States. USDA:APHIS:VS, CEAH, Fort Collins, CO.

van der Linde, C., G. de Jong, E. P. Koenen, and H. Eding. 2010. Claw health index for Dutch dairy cattle based on claw trimming and conformation data. J. Dairy Sci. 93:4883-4891.

Wilshire, J. A., and N. J. Bell. 2009. An economic review of cattle lameness. Cattle Pract. 17:136-141. 